\title{
THE GLOBAL DISTRIBUTION OF ZODIACAL DUST
}

\author{
B. Kneißel \\ Bereich Extraterrestriche Physik \\ Ruhr-Universität Bochum \\ D-4630 Bochum FRG
}

\begin{abstract}
From visible or infrared brightness observations of the zodiacal light, a large variety of models of the three-dimensional structure of the zodiacal dust cloud has been proposed. To assess the reliability of these models, we must first investigate their fit to a selected set of observational data. The fit is best for bulge models, which have an appreciable density over the solar poles. Next, we check the orbital inclination distributions predicted by the various models. A comparison of these distributions with those of minor bodies in the solar system does not support the preference for bulge models, but instead supports polar hole models with a negligible density over the solar pole. These uncertainties of modelling have to be kept in mind when models are used to derive the brightness contribution of zodiacal light, particularly in the infrared, where the data base is still limited.
\end{abstract}

\section{PHOTOMETRY AND MODELS OF SPATIAL DISTRIBUTION}

For each of the proposed three-dimensional models of the interplanetary dust distribution, we compute the predicted brightness $Z_{\text {calc }}$ according to the procedures of Giese, Kneißel, and Rittich (1986) and Giese and Kneißel (1989). Albedo changes with heliocentric distance, as indicated from IRAS observations, are taken into account. The observed brightness values $Z_{\text {obs }}$ of Levasseur-Regourd and Dumont (1980) are taken for comparison. We use the relative deviation $\left(Z_{c a l c}-Z_{o b s}\right) / Z_{o b s}$ as a measure of the quality of the model.

Among the classical models (Giese, Kneißel, and Rittich, 1986), the flat ellipsoid model (Dumont, 1976a) gives a good fit to observations in directions opposite to the sun (about $-15 \%)$. But in directions close to the sun, observational values are underestimated (less than $-20 \%$ ). In contrast, the broad fan model (Leinert et al., 1981), which has been made to fit near-ecliptic data from HELIOS, compares well with the brightness observed near the sun, whereas the polar brightness is too large by $>30 \%$. To improve the fit to observations taken in sunward viewing directions in the flat ellipsoid model, Dumont added a bulge of spherical shape at the solar poles, leading to his sombrero model (Dumont, 1976b). The same is true for a fan model by Lumme and Bowell (1985) and for the "optimal sombrero" (Giese, Kneißel, and Rittich, 1986). These models fit the data to better than 20\%. Multilobe models, such as those proposed by Buitrago, Gomez, and Sanchez, (1983) do not show a steady decrease of density with helioecliptical latitude, as all the others do. Although such multilobe distributions appear highly improbable, it is only for viewing close to the sun that they do not fit the data. Models from infrared data, including the model by Lamy and Perrin (1986), have low density above the solar poles (polar hole models). Their fit to the observations far from the sun is reasonable. For observations close to the sun, these models underestimate the brightness by $\geq 40 \%$. Thus, bulge models are favored as long as we discuss only the brightness distribution. 


\section{DYNAMICS WITH EMPHASIS ON THE INCLINATION DISTRIBUTION}

To allow a more physical discussion, distributions of orbital elements were derived from the different three-dimensional models. In particular, the distribution of inclinations places important restrictions on the three-dimensional distribution of zodiacal dust.

The number of particles in orbits of inclination $i$ determines a minimum number density for helioecliptical latitudes less than $i$, according to their probability of being found there. Multilobe models violate this minimum number density condition.

When the inclination distributions derived from the various three-dimensional models are compared, the flat ellipsoid model shows a maximum very close to the ecliptic (at $6^{\circ}$ ), whereas the broad fan model has its maximum at about $15^{\circ}$. None of the distributions resembles the distribution from micrometeoroids (Andreev and Belcovich, 1985), which are thought to be the source for the zodiacal dust.

The isotropic background component in the inclination distribution of bulge models suggests that part of the dust should be formed by particles in retrograde orbits. Theoretical considerations, however, show that the input into the zodiacal cloud from meteoroids of retrograde motion by fragmentation is very unlikely because the eccentricities of mother particles are appreciably high. Also, micrometeoroid impact experiments have not yet detected this type of particle (Grün et al., 1980). Finally, from the detection of Doppler-shifted Fraunhofer lines in the zodiacal light, James and Smeethe (1970), in agreement with others, estimate the fraction of particles in retrograde orbits to be $5 \%$ at maximum. Bulge models, with their large isotropic component, exceed this limit, whereas the polar hole models give an adequate solution and are preferred on these grounds.

\section{DISCUSSION}

Present three-dimensional models show substantial differences in the shape of the zodiacal cloud and in the absolute number densities of dust particles per cubic meter at the earth's orbit. This lack of a reliable model for the spatial distribution of interplanetary dust means that one has to be very careful when using such models to estimate the brightness contribution of zodiacal light.

Acknowledgments - These investigations have been inspired and encouraged by the late Professor Dr. R. $\mathrm{H}$. Giese. This work was supported by the Bundesminster für Forschung und Technologie (Contract 01-0N-014-2).

\section{REFERENCES}

Andreev, V. V., and Belcovich, O. I. 1985, presented at IAU General Assembly (New Delhi). Buitrago, J., Gomez, R., and Sanchez, F. 1983, Planet. Space Sci., 31, 373.

Dumont, R. 1976a, Lecture Notes in Physics, 48, 85.

Dumont, R. 1976b, presented at the IAU General Assembly, Grenoble.

Giese, R. H., and Kneißel, B. 1989, ICARUS, in press.

Giese, R. H., Kneißel, B., and Rittich, U. 1986, ICARUS, 68, 395.

Grün, E., et al. 1980, Planet. Space Sci., 28, 333.

James, J. F., and Smeethe, M. J. 1970, Nature, 227, 588.

Lamy, P. L., and Perrin, J.-M. 1986, Astron. Astrophys., 163, 269.

Leinert, Ch., Richter, I., Pibz, E., and Planels, B. 1981, Astron. Astrophys., 103, 77.

Levasseur-Regourd, A. C., and Dumont, R. 1980, Astron. Astrophys., 84, 277.

Lumme, K., and Bowell, E. 1985, ICARUS, 62, 54. 